Copyright (C) 2013 IEEE. Personal use of this material is permitted. Permission from IEEE must be obtained for all other uses, in any current or future media, including reprinting/republishing this material for advertising or promotional purposes, creating new collective works, for resale or redistribution to servers or lists, or reuse of any copyrighted component of this work in other works. 


\title{
Digital Channel Capacity Calculation Using Augmented Ordered Binary Decision Diagrams
}

\author{
Johannes U. Herrmann and Sieteng Soh \\ Department of Computing \\ Curtin University \\ Bentley, Western Australia \\ iherrmann@ieee.org
}

\begin{abstract}
Digital channel capacity of a network is an important factor in network design. Recent solutions use the Markov chain to model and compute the capacity of only a specific wireless network with multiple relays and multiple hops (MR-MH), and thus cannot be used for networks in general. Further, the solutions assume that all communication link failure probabilities are equal and that state transitional probabilities are known. This paper proposes a general solution for computing the capacity. Our solution first converts the problem of computing the channel capacity of such networks into a network reliability problem. Then, it applies the Augmented Ordered Binary Decision Diagram (OBDD-A) method to compute the reliability, and hence the channel capacity. The OBDD-A does not require the aforementioned assumptions, and can be applied to a wide range of networks. Since the OBDD-A has been shown to have linear time complexity and constant space complexity for families of networks with constant inter-connectivity (such as MR-MH) the proposed solution is extremely efficient. The application of our method is verified by simulations.
\end{abstract}

Index Terms-channel capacity, multi-relay networks, digital channel modeling, augmented ordered binary decision diagram, network reliability.

\section{INTRODUCTION}

Wireless communications networks are becoming prevalent in the developed world, and therefore they must be optimally designed to meet application demands. A number of issues affect the performance of wireless networks, including reduction of signal at increasing distance from base stations, energy efficiency and communication range [1]. These difficulties have been addressed, among others, by using networks with multiple relays, each possibly having multiple hops. Adding additional hops to a relay decreases the distance of each wireless communication, and hence increases the likelihood of successful communication in that hop while decreasing the power needed to send the message [1]. The addition of hops, however, also increases the number of places where a communication error can occur [2]. In contrast, adding additional relays increases the chance that at least one correct communication will occur. The multi-relaymulti-hop networks are referred to as MR-MH networks; and example is shown in Fig 1.

The network's ability to channel data at a suitable capacity is also an important issue in the design and implementation of networks, including wireless networks. The capacity is affected by packet loss, in which case the receiving node (e.g., in the Internet) will detect a checksum or check bits error and request packet retransmission. The speed at which data is transmitted through a wireless network is affected by the channel capacity, and is seen as the inherent limiting factor to performance in current and future systems [3].

The computation of the capacity of MR-MH networks has been addressed by Berber, Kovacevik and Temerinac $[4,5]$ using a multi-state model. Markov chains are used to find steady state probabilities at any given time period, and these, in turn, are used to compute channel capacity. This work assumes that communication channels have memory and that a decode-and-forward relay strategy is used [5] The channel capacity is the greatest possible rate for the channel, where rate in bits per transmission is defined in [6].

The paper [4] shows how to derive the Markov chain to compute the capacity for small networks that contain only one or two relays. While the method can be extended to networks with a larger amount of relays, each such formula must be calculated independently. Further, each formula contains multiple summations and products of conditional probabilities, and thus producing the formulas by hand is time consuming, if feasible, for larger networks. Further, while the method [7] is able to produce a general formula for MR-MH networks of arbitrary size, the formula is only applicable to such networks that have equal communication link failure probabilities. Finally, all formulas presented only

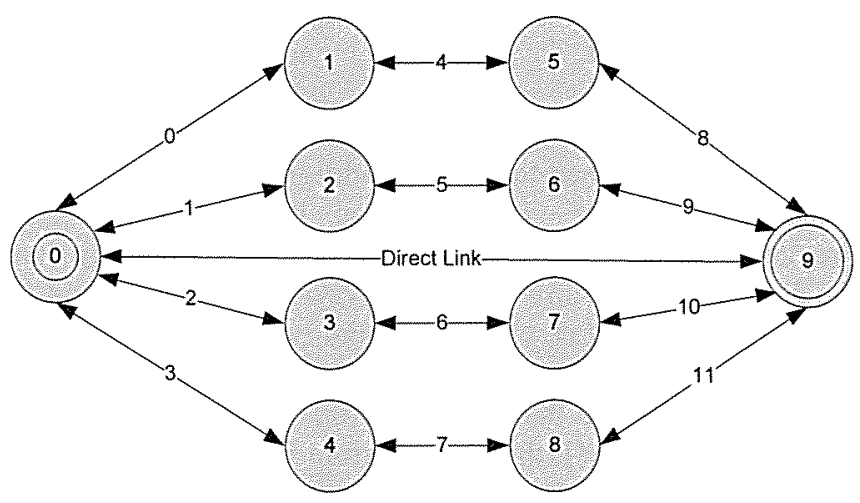

Figure 1. Sample MR-MH Network 
apply to MR-MH models and not to other relevant network models.

Renk, Iankov and Jondral [8] also considered the capacity of MR-MH networks but approached it as an optimization problem and computed bounds rather than the actual capacity value. Further, most of the work addressed networks with either relays or multiple hops and the case of both was seen as simply the maximal bound of both individual cases.

In this paper, we propose using Augmented Ordered Binary Decision Diagrams (OBDD-A) to automatically analyze the channel capacity of networks with a potentially large number of relays and hops. Specifically, the main contribution of this paper is twofold. First, we show how to convert the problem of computing the capacity of MR-MH networks in $[4,5]$ into a network reliability problem, defined in Section III. We then show how to solve the reliability problem, and hence the MR-MH problem, using OBDD-A. Our simulations, discussed in Section IV, show that OBDD$\mathrm{A}$ is an appropriate tool for computing the channel capacity of MR-MH networks of even large size, and is equally applicable to many other networks.

The remainder of the paper is organized as follows. In Section II we review network capacity as defined in $[4,5]$, the concept of network reliability and the use of the OBDD-A to compute reliability. In Section III we demonstrate the use of OBDD-A for computing the capacity of a MR-MH network. Section IV presents results of a number of tests, and discusses the outcomes, and Section V concludes the paper.

\section{BACKGROUND}

This section briefly convers the three areas of background knowledge relevant to this paper. First we consider the concept of network capacity as defined in $[4,5]$. We then outline the concept of network reliability and the OBDD-A method used to compute reliability and other metrics.

\section{A. Network Capacity}

A MR-MH network [4] has one direct connection between given source $(s)$ and target $(t)$ nodes, and a number of relay connections, each possibly having multiple hops; Fig. 1 shows an example network with $s=0, t=9$ and four relay connections. In such network, a communication is successful only if the direct communication link between the source and target is successful, and if at least one communication passes along a relay successfully.

The authors [4] use Markov Chains in their method, and thus almost all probabilities are conditional. For example the probability that the communication fails given that the previous one failed is denoted $\mathrm{P}(0 \mid 0)$ and the probability that the communication fails given the previous one succeeded is denoted $P(0 \mid 1)$. Let $P(0)$ be the probability of the communication failing and $P(1)$ be the probability that it succeeds. It is noted that $\mathrm{P}(0 \mid 0)=\mathrm{P}(0 \mid 1)$, and hence both are also equal to $P(0)$ [4]; this follows from the chance of packet failure being independent of the success of previous packets. Similarly, $P(1 \mid 0)=P(1 \mid 1)=P(1)$. This indicates that state probabilities $P(0)$ and $P(1)$ can be used instead of the conditional probabilities.

Markov chains are used to find formulaic expressions for $P(0)$ and $P(1)$, which are then combined to give the channel capacity, $C[9]$, defined as

$$
C=1+\mathrm{P}(0) \log _{2} \mathrm{P}(0)+\mathrm{P}(1) \log _{2} \mathrm{P}(1) \text {. }
$$

Finally, a general formula for a network of $n$ relays is derived as

$C=1+B L_{e} \times \log _{2} B L_{e}+\left(1-B L_{e}\right) \times \log _{2}\left(1-B L_{e}\right)$

for

$$
\begin{aligned}
B L_{e}=[1- & \left.(1-B L)^{H}\right]^{2} \times B L_{\bar{c}}+ \\
& \left(1-\left[1-(1-B L)^{H}\right]^{2}\right) \times B L_{C},
\end{aligned}
$$

where each communication link has an equal probability of packet error, $B L$, and $B L_{C}$ and $B L_{\bar{c}}$ are the packet error rates in the co-operative and non-co-operative state, respectively, and $H$ is the number of hops in the network. Note that this general formula cannot be calculated using the given method if the packet error rates are not identical. In addition, both $B L_{C}$ and $B L_{\bar{C}}$ have to be computed. Further, the formula can be used only for networks of the type described, but not for any other type of network.

For the MR-MH network shown in Fig. 1 with all communications having a failure rate of $0.01, P(0)$ is computed to be 0.010001 and $P(1)$ is 0.989999 . Hence, the capacity for this network is 0.919202 .

\section{B. Network Reliability}

The reliability of a network is defined as the probability that it is connected. In other words, it is the probability that a specified communication passing through all or parts of the network is successful.

A number of different reliability models exist $[10,11]$. For example, 2-terminal reliability is when the communication is between a single source and a single target (destination) device in the network. If the communication is between a set of $K$ devices in the network, $K$-terminal reliability applies. When communication is required between all nodes in the network, the all-terminal reliability model is used. A number of more general network reliability models also exist, such as a network with multiple source and target devices requiring a connection between at least one source and one target.

Further, reliability may be applied to different network models. A network may have fallible communication links between devices but devices which, themselves, do not fail. Wireless sensor networks are often modelled as networks whose devices may fail (due to power loss, etc.) but whose communication links are infallible. Finally, a network may have both fallible devices and communication links.

When computing network reliability, it is usual to represent the devices and communication links of a network by the vertices and edges of a graph. Each edge $e_{i}$ of the network is assigned a probability $0 \leq P\left(e_{i}\right) \leq 1.0$ of being available. It is assumed that all edges fail independently. 


\section{Augmented Ordered Binary Decision Diagrams}

Numerous methods exist for computing the reliability of a network. While most approaches apply to only one or two reliability and network models, the Augmented Ordered Binary Decision Diagram (OBDD-A) $[11,12]$ is applicable to a wide range of networks and communications. Further the OBDD-A is capable of analysing very large networks, such as the $4 \times 40,000$ grid network. Finally, the OBDD-A is capable of computing metrics such as expected hop count [11] and message delay [12]. The original OBDD-A has been combined with the boundary set method [13] to give the hybrid OBDD-A, which is very efficient on undirected networks.

The OBDD-A method builds a binary decision diagram [14] with augmented nodes that store data relevant to the metric being computed. For example, for network reliability each node stores a state probability and for hop count each node stores the hop counts to currently reached devices.

Each node represents a network state. When a state represents a state of the network where communication is successful, the information in the node is recorded and the node is discarded. A node representing a state in which the communication can no longer be successful is immediately discarded. All nodes that fit neither of these categories are iteratively processed to generate child nodes, each of which represents a network state. The process continues until all nodes represent either successful or failure states $[11,12,15]$.

The performance of the OBDD-A has been shown to be related to the width of the network rather than its length [15]. Further, it has been shown that for families of networks of constant width (such as all MR-MH networks with a fixed number of relays) the OBDD-A has linear time performance and constant space performance.

\section{USING THE OBDD-A TO COMPUTE CHANNEL CAPACITY}

\section{A. Reliability of MR-MH Networks}

A communication in a MR-MH network is successful if the information sent through the direct connection is correctly received and if at least one relay also delivers an error-free communication. The probability that a packet is received successfully by a node is analogous to the reliability concept of edge failure; if the edge is available the packet is received

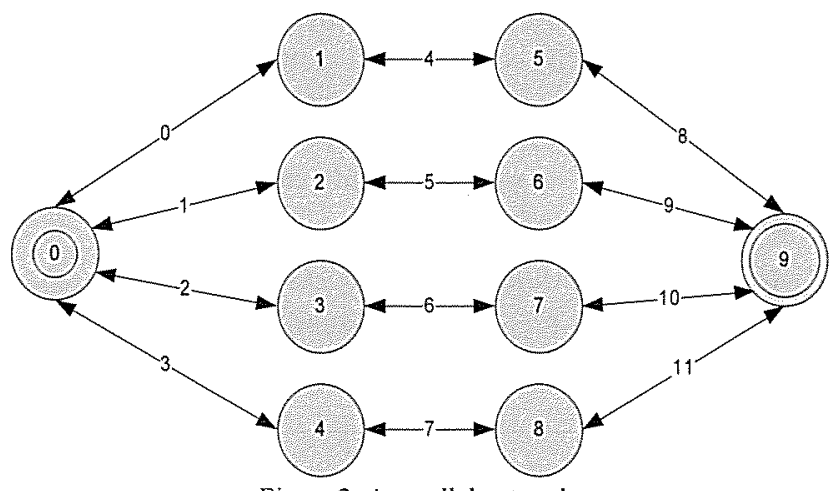

Figure 2. A parallel network correctly and if the edge is failed the packet has an error. Hence the error rate $\mathrm{BL}$ from $[4,5]$ is translated into the edge probability $\mathrm{P}\left(\mathrm{e}_{\mathrm{BL}}\right)$ by using $\mathrm{P}\left(\mathrm{e}_{\mathrm{BL}}\right)=1-\mathrm{BL}$ where $\mathrm{e}_{\mathrm{BL}}$ is the edge that the packet corresponding to $\mathrm{BL}$ travels along.

With this transformation, the reliability of the MR-MH network becomes equivalent to computing $P(1)$, and the unreliability ( 1 - reliability) is equivalent to $P(0)$. Hence computing the reliability of the MR-MH is sufficient for computing $C$.

However, the definition of a successful communication in a MR-MH network does not fit into any of the standard models of reliability because a specific path (represented by a single edge from source to destination) is singled out as a requirement. Therefore, another transformation, described below, must be applied in order to use existing reliability tools such as the OBDD-A $[11,12]$.

Label the source vertex $v_{0}$ and the target (destination) vertex $v_{t}$ and the edge that connects them directly $\mathrm{e}_{0}$. Label all other vertices and edges in the network in order of increasing distance (number of hops) from the source. Such an ordering [11] can be seen in the graph in Fig 2 .

We now decompose the graph representing the given MR-MH network $G$ into sub-graphs $G^{\prime}=\left(V, E-\left\{e_{0}\right\}\right)$ and $G^{\prime \prime}=\left(\left\{v_{0}, v_{t}\right\},\left\{e_{0}\right\}\right)$, where $e_{0}$ is the edge connecting the source and sink vertices directly. The sub-graph $G^{\prime}$ corresponding to the MR-MH network in Fig. 1 is shown in Fig. 2. We can compute the probability of successful communication in G using G' and G"; the communications in both sub-graphs are required to succeed in order for the communication in $\mathrm{G}$ to be successful. However, sub-graphs G' and G' are edge-disjoint, meaning the probability of both having successful communications is equal to the product of their individual two-terminal reliabilities.

1. Decompose the graph $\mathrm{G}$ into
a. $\mathrm{G}^{\prime}=\left(\mathrm{V}, \mathrm{E}-\left\{\mathrm{e}_{0}\right\}\right)$, and
b. $\mathrm{G}^{\prime \prime}=\left(\left\{\mathrm{v}_{0}, \mathrm{v}_{\mathrm{t}}\right\},\left\{\mathrm{e}_{0}\right\}\right)$
2. Use OBDD-A to compute $\operatorname{REL}\left(\mathrm{G}^{\prime}\right)$
3. Compute $\operatorname{REL}\left(\mathrm{G}^{\prime \prime}\right)=\operatorname{Pr}\left(\mathrm{v}_{0}\right) \times \operatorname{Pr}\left(\mathrm{e}_{0}\right) \times \operatorname{Pr}\left(\mathrm{v}_{\mathrm{t}}\right)$
4. Compute REL $(\mathrm{G})=\operatorname{REL}\left(\mathrm{G}^{\prime}\right) \times \operatorname{REL}\left(\mathrm{G}^{\prime \prime}\right)$
5. $\mathrm{P}(1)=\operatorname{REL}(\mathrm{G})$ and $\mathrm{P}(0)=1-\operatorname{REL}(\mathrm{G})$
6. $C=1+\mathrm{P}(0) \log _{2} \mathrm{P}(0)+\mathrm{P}(1) \log _{2} \mathrm{P}(1)$

Figure 3. Algorithm to Compute Capacity from Reliability

The reliability of G', REL(G'), is $P\left(e_{0}\right)$ and $\operatorname{REL}\left(G^{\prime}\right)$ can be computed using the OBDD-A method for two terminal reliability. From these reliabilities it is possible to form $\mathrm{P}(1)$ $=\operatorname{REL}\left(\mathrm{G}^{\prime}\right) \times \operatorname{REL}\left(\mathrm{G}^{\prime \prime}\right)$ and $\mathrm{P}(0)=1-\mathrm{P}(1)$ and hence compute $C$. The process is summarized in Fig. 3.

\section{B. The Reliability of Parallel Networks}

The G' portion of an MR-MH is a series of parallel relays with multiple hops, referred to as a parallel (or disjoint) network. One such network, with four relays and two hops, can be seen in Fig. 2. Given that the type of network affects the performance of the algorithm computing reliability, it is 
important to discuss the suitability of the OBDD-A as a tool for computing REL(G').

The efficiency of the OBDD-A has been shown to be linked to $F_{m a x}$, the maximum size of the boundary set [15]. This makes the OBDD-A especially efficient on networks that have low width. Define the width of $G^{\prime}$ as

$\operatorname{MAX}\left(|j-i|:\left(\mathrm{v}_{i}, \mathrm{v}_{j}\right)\right.$ is an edge of $\left.\mathrm{G}^{\prime}\right)$,

assuming that the edges and vertices of $G^{\prime}$ are ordered as described in section III A.

It can be seen that the width of the network in Fig. 2 is four, which corresponds to the intuitive meaning of width if we count the number of hops as 'length'. Increasing the number of hops per relay does not increase the width; the width (and hence $F_{\max }$ ) is dependent entirely on the number of relays. OBDD-A has been shown to work well with networks of reasonably small width $(<10)$. For example, the OBDD-A computes the two terminal reliability of the $7 \times 1,000$ grid network in 93.5 seconds while requiring only 21.8 seconds for the $4 \times 40,000$ grid network (as shown in Section IV.

In addition, the OBDD-A performs better for networks with a lower number of edges. For example the worst-case performance of the OBDD-A is on fully-connected networks where each vertex is connected to every other vertex. For example, the OBDD-A requires 0.05 seconds to compute the two terminal reliability of the $3 \times 4$ grid of 12 vertices, but requires more than 26 minutes for the fully connected network of 12 vertices. Parallel networks have a relatively low number of edges, and thus are suitable for analysis by the OBDD-A

The performance of the implemented OBDD-A on a range of parallel networks is discussed in Section IV.

\section{Computing the Channel Capacity of Other Networks}

While the MR-MH model is suitable for extending a wireless channel, it is not the only possible model. In particular, the nodes of a wireless sensor network may be randomly spread over an area or it may be useful to cross-link channels to improve the overall reliability of the system either with the nodes on the next level the neighbouring relays (as shown in Fig. 4) or the same level (as shown in Fig.

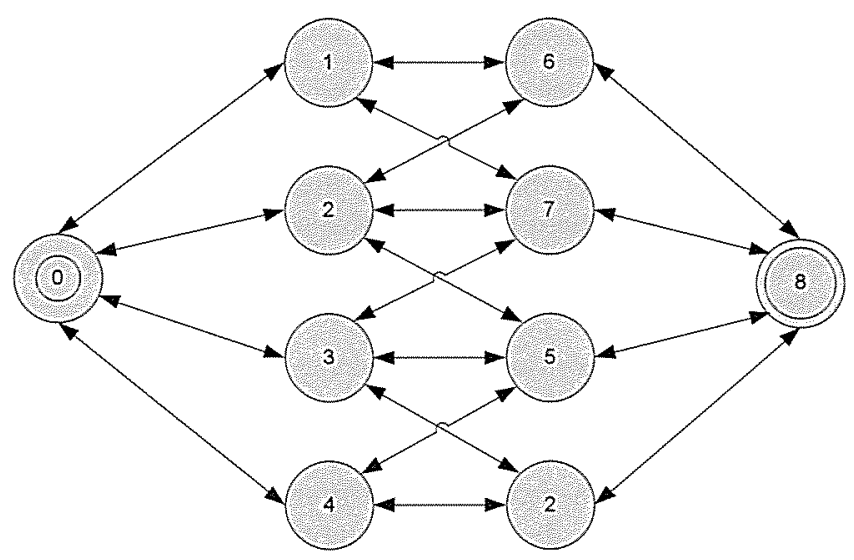

Figure 4. An interlinked network

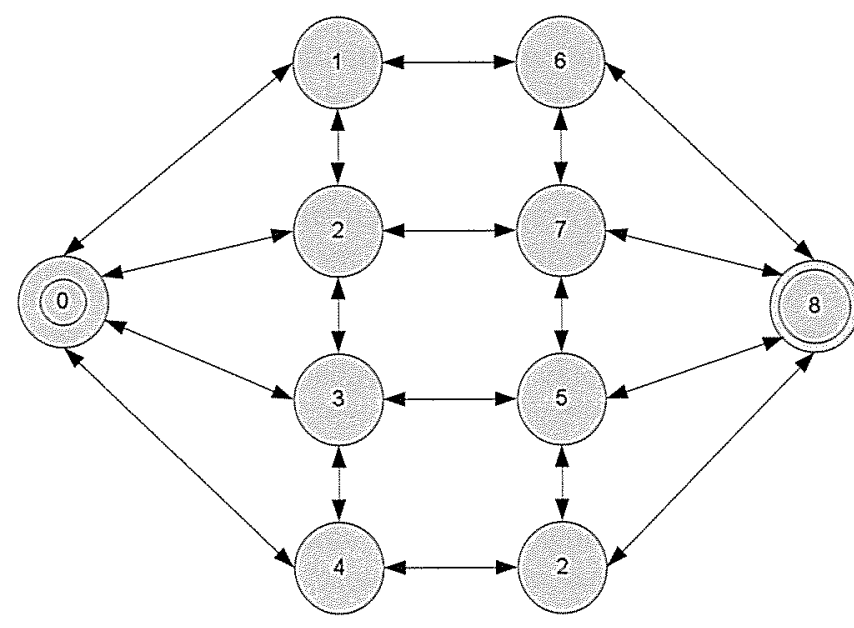

Figure 5. A grid-linked network

5). It should be noted that the latter grid pattern simulates the capability of wireless nodes to communicate with all neighbouring nodes.

While it is difficult to calculate a general formula to handle any form of network, generic tools like the OBDD-A can be used to compute the network reliability of networks of almost any configuration. This reliability can, in turn, be used to computing the digital channel capacity.

In addition to computing the channel capacity, the network reliability itself can be of use to network designers. In addition, the OBDD-A can also be used to compute other metrics such as the expected hop count [11] and message delay [12]. These provide further information for designers, especially if the network has power considerations or requires delay guarantees.

\section{EXPERIMENTAL RESULTS}

Each algorithm was implemented using Microsoft Visual Studio 2010 on a PC (i7 $9202.67 \mathrm{GHz}$ processors, 8MB L3 cache, 12GB RAM) running Windows 7. Networks were generated by Perl scripts and pre-sorted by another Perl script that implements the ordering described in Section III. For each simulation, the run time, in CPU seconds, was averaged over five runs, which was deemed to be sufficient due to the low variance'.

The simulations assumed a communication link failure rate of 0.1 , which is the most commonly used rate in network reliability literature. It is assumed that graph vertices (and thus the network devices) do not fail. The algorithm allows each edge and vertex to be assigned individual failure rates if required.

\section{A. Multiple-Relay Multi-Hop Networks}

A number of parallel networks were generated, representing the second part of the separated MR-MH

\footnotetext{
1 The greatest variance for the results presented in this paper was 0.002 for the 4 relay, 3000 hop network.
} 
network. The OBDD-A was able to compute the answer to a network of three relays, each of five hops, in 0.033 seconds. A somewhat more robust network, with four relays of 20 hops each, required 0.037 seconds. A large network of five relays of 100 hops each required 0.078 seconds. In general, MR-MH networks of a size far larger than those discussed in $[4,5]$ can be solved in a fraction of a second.

It can thus be seen that computing the reliability of a parallel network of even large size is extremely fast using the OBDD-A algorithm. As long as the number of relays is relatively small, this performance continues to be excellent.

For example, consider a series of networks of four relays, with an increasing number of hops per relay. The processing time increases linearly with the number of hops, as shown in Fig. 6. Unless an unusually large number of relays is required, the linear complexity guarantees that the OBDD-A can compute the reliability of a parallel network in a reasonable amount of time.

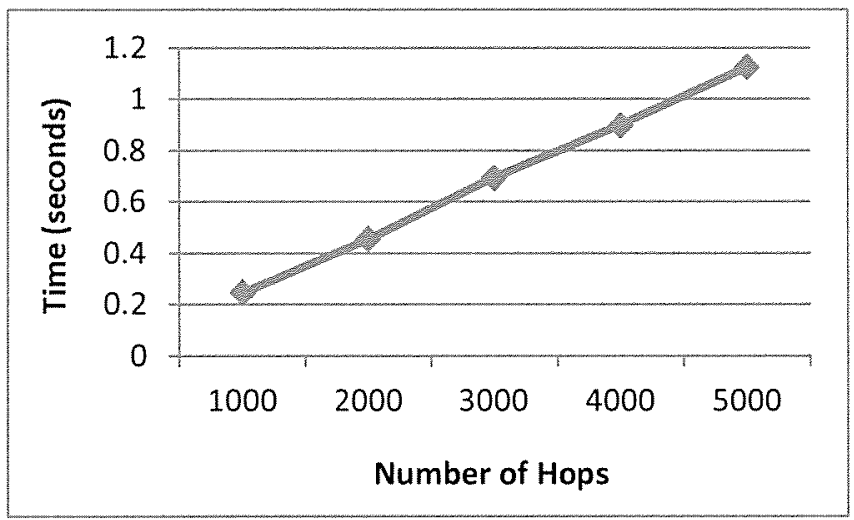

Figure 6. Relationship between hops and time in a 4 relay network.

This linear time complexity for families of networks with a constant number of relays has been previously shown to be true for the OBDD-A [15]. In addition, the space performance for such a family of networks is constant. For example, each of the networks in Fig. 6 required at most 15 diagram nodes to be stored in memory at any one time.

It is thus clear that the OBDD-A is a suitable tool for computing the reliability of parallel networks, and hence the channel capacity of MR-MH networks. However, since the OBDD-A is a general tool; it can also be used to compute the channel capacity of other types of networks. Further, it can compute metrics other than just reliability.

\section{B. Other Networks and Metrics}

The OBDD-A has been shown $[11,12]$ to be able to compute the reliability, hop count and message delay for a wide range of networks.

Let the width of a network be $\operatorname{MAX}\left(\left|\mathrm{v}_{f}-\mathrm{v}_{f}\right|\right.$ for any pair of vertices $v_{f}$ and $v_{t}$ that are connected by an edge. Note that width is related to the network's diameter [3]. Networks with low width such a parallel and grid networks are especially suitable for analysis with an OBDD-A. For example, the OBDD-A can compute the two terminal reliability of the $4 \times 40,000$ grid network in 21 seconds and the two terminal reliability of the $7 \times 1,000$ grid network in 104 seconds.

Interconnectivity also affects the performance of the OBDD-A since it increases the width measure of the network. For example a parallel network and grid network with the same number of relays and hops have the same width, but a cross-connected network of the same size has greater width. This results in poorer performance.

For example the cross-connected network with five relays and 100 hops requires 4 seconds as compared to 0.113 seconds for the comparable parallel network. Similarly, the cross-connected network with four relays and 5000 hops requires 21.3 seconds as compared to 1.1 seconds for the corresponding parallel network. While the OBDD-A method can still be used to solve such networks, it is best to avoid networks with a large amount of relays.

The OBDD-A can also be used to compute the expected hop count (EHC) [11] and expected message delay (EMD) [12] of networks. Due to the additional computation involved, this requires more time and space than computing network reliability. For example, computing the two terminal $\mathrm{EHC}$ for the parallel network with 5 relays and one hundred hops in 0.083 seconds while the network with 5 relays and one thousand hops requires 0.295 seconds.

\section{Digital Channel Capacity and Component Reliability}

Berber, Kovacevik and Temerinac $[4,5]$ show that the channel capacity is directly related to the reliability probability of each part of the network, for the case where all packet error probabilities are equal. The OBDD-A implementation generated matching results.

Table 1 shows the digital channel capacity of three networks at different packet error rates, under the assumption that each component of the network has identical error rates. The three networks tested were the parallel networks with 4 relays and 2 hops ( $\mathrm{R} 4 \mathrm{H} 2$ ), and 3 relays and 5 hops ( $\mathrm{R} 3 \mathrm{H} 5$ ). The $3 \times 100$ grid network was also tested.

TABLE I. RELATIONSHIP BETWEEN CHANNEL CAPACITY AND COMPONENT RELIABILITY

\begin{tabular}{|cc|c|c|}
\hline Prob & R4 H2 & R3 H5 & Grid 3×100 \\
0.9 & 0.515803 & 0.293154 & 0.210955 \\
0.99 & 0.919202 & 0.917894 & 0.917199 \\
0.999 & 0.988592 & 0.988590 & 0.988571 \\
\hline
\end{tabular}




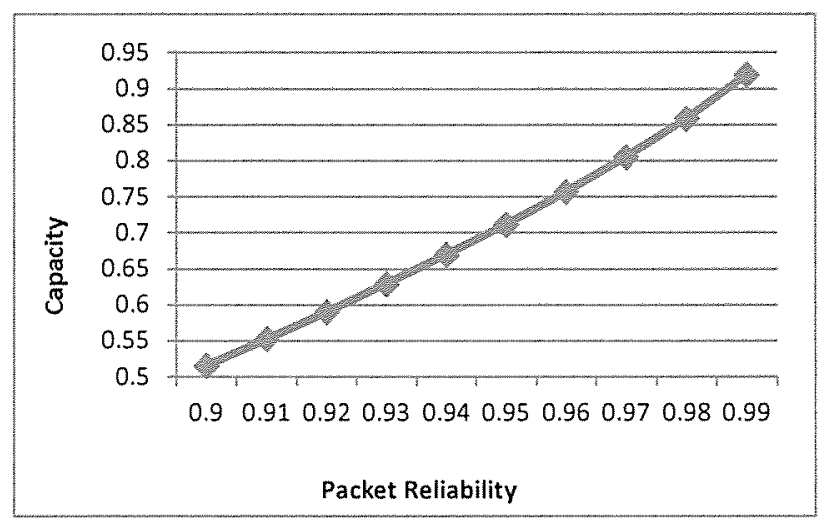

Figure 7. Relationship between packet reliability and capacity

The results from Table 1 show that increasing the number of hops of a network decreases the reliability (and hence the capacity) of a network. The probability of packet success (or packet reliability) directly affects the capacity, as shown in both Table I and Fig. 7. The latter shows the capacities for the R4 H2 MR-MH network shown in Fig. 1.

\section{CONCLUSIONS}

In this paper, we have demonstrated that the OBDD-A can be used to efficiently compute the digital channel capacity of wireless MR-MH networks. While the original method $[4,5]$ requires the manual computation of a large number of factors for even small graphs, the OBDD-A can compute the reliability of extremely large MR-MH networks in seconds

Furthermore, the OBDD-A does not require that the network be restricted to having equal communication link failures.

In addition, the OBDD-A has been shown to be of use for computing the digital channel capacity for networks other than MR-MH networks, and indeed for computing a range of other metrics on these networks. However the OBDD-A does always not scale well for general networks with large width. More research is required to determine if the OBDD-A is appropriate for computing the digital channel capacity of practical networks with hundreds of devices.

We thus conclude that the OBDD-A is an appropriate tool for computing the digital channel capacity of MR-MH networks and may prove useful for general networks.

\section{REFERENCES}

[1] W. S. Akyildiz, W. Su, Y. Sankarasubramaniam, and R. Cayirci "Wireless sensor networks: a survey," Computer Networks, vol 38, pp. 393-422, March 2002

[2] I. F. Akyildiz, X. Wang, and W. Wang, "Wireless mesh networks: a survey," Computer Networks, vol. 47 , pp. 445-487, Mar. 2005.

[3] D. B. Johnson, D. A. Maltz, and J. Broch, "DSR: The dynamic source routing protocol for multi-hop wireless ad hoc networks," Ad hoc networking, vol. 5, pp. 139-172, 2001.

[4] S. Berber, V. Kovacevic, and M. Temerinac, "Digital channel modeling and capacity calculation for multi-relay multi-hop wireless sensor networks," in $5^{\text {th }}$ WSEAS International Conference on Communications and Information Technology 2011.

[5] S. Berber, V. Kovacevic, and M. Temerinac, "Digital channel modeling for multi-relay wireless sensor networks," in $5^{\text {th }}$ WSEAS International Conference on Commmications and Information Technology 2011.

[6] T. Cover and A. E. Gamal, "Capacity theorems for the relay channel," IEEE Trans. Information Theory, vol. 25, pp. 572-584, 1979.

[7] J. Ciric, C. Sechen, and -. N. P. s.- 617, "Efficient canonical form for Boolean matching of complex functions in large libraries," in IEEE/ACM Intlernational Conference on Computer Aided Design (ICCAD 2001), 2001.

[8] T. Renk, D. Iankov, and F. K. Jondral, "Adaptive Resource Allocation in Wireless Relay Networks," in Vehicular Technology Conference, 2009. VTC Spring 2009. IEEE 69th, 2009 , pp. 1-5.

[9] E. N. Gilbert, "Capacity of a burst-noise channel," Bell Syst. Tech. J, vol. 39, pp. 1253-1265, 1960.

[10] F. Beichelt, "Reliability Preserving Network Transformation Applications and Problems," International Journal of Reliability, Quality and Safety Engineering, vol. 14, pp. 189-199, 2007.

[11] J. U. Herrmann. S. Soh, S. Rai, and G. West, "On Augmented OBDD and Performability for Sensor Networks," International Jounal of Performability Engineering, vol. 6, pp. $331-342$, Jul. 2010.

[12] J. U. Herrmann, S. Soh, S. Rai, and M. Škorjanc, "Computing Performability for Wireless Sensor Networks," International Joumal of Performability Engineering, vol. 8, pp. 131-140, March 2012.

[13] G. Hardy, C. Lucet, and N. Limnios, "K-Terminal Network Reliability Measures With Binary Decision Diagrams," IEEE Transactions on Reliability, vol. 56, pp. $506-515$, Sept. 2007.

[14] R. E. Bryant, "Symbolic Boolean Manipulation with Ordered Binary-Decision Diagrans," ACM Computing Strveys, vol. 24 , pp. 293-318, 1992.

[15] J. U. Hermann and S. Soh, "A Memory Efficient Algorithm for Network Reliability," in $15^{\text {th }}$ Asia-Pacific Conference on Communications (APCC2009), 2009 